\title{
Clinically feasible method for assessing leukocyte rheology in whole blood
}

\author{
Riha Shimizu ${ }^{1} \cdot$ Hirotsugu Fukuda ${ }^{1,2} \cdot$ Yuji Kikuchi $^{3} \cdot$ Hirokazu Yanaka $^{4} \cdot$ Nobuhiro Hata $^{5} \cdot$ Masashi Yamazaki $^{5}$. \\ Yuki Nakatani $^{6} \cdot$ Yuma Tamura $^{7} \cdot$ Seiko Yamakoshi $^{7} \cdot$ Atsuhiko Kawabe $^{8} \cdot$ Yasuto Horie $^{9} \cdot$ Hiroyuki Sugimura $^{9}$. \\ Yasushi Matsushita ${ }^{1} \cdot$ Takaaki Nakamoto $^{9} \cdot$ Takanori Yasu $^{8}$
}

Received: 7 May 2019 / Accepted: 16 August 2019 / Published online: 23 August 2019

(c) The Author(s) 2019

\begin{abstract}
This study reports a novel method for assessment of leukocyte rheological activation with a new designed microchannel array chip to mimic the human microvascular network for microchannel array flow analysis (MCFAN). Study subjects were 79 healthy volunteers and 42 patients with type 2 diabetes mellitus (DM) and 36 patients with acute coronary syndrome (ACS). Using the anticoagulants heparin and ethylene-diamine-tetraacetic acid (EDTA)-2Na which inhibits platelets and leukocytes by chelating $\mathrm{Ca}^{2+}$, we were able to quantify leukocyte rheological activation by the subtraction of passage time of blood treated with both heparin and EDTA-2Na from that of blood treated with heparin only. We confirmed that passage times of whole blood with heparin + EDTA-2Na were always shorter than those of whole blood with only heparin in healthy subjects and patients with DM or ACS under suction pressures of $-30 \mathrm{~cm} \mathrm{H}_{2} \mathrm{O}$. There was a significant correlation between delta whole blood passage time $\{$ (heparin tube) - (EDTA-2Na + heparin) $\}$ and serum levels of myeloperoxidase and adhesive leukocyte number, respectively, even in blood from patients with DM or ACS, who suffered from inflammation. In conclusion we have developed a clinically feasible method for assessing leukocyte rheological activation in whole blood in ex vivo.
\end{abstract}

Keywords Acute coronary syndrome $\cdot$ Diabetes mellitus $\cdot$ Leukocyte $\cdot$ Microcirculation $\cdot$ Rheology

\section{Introduction}

Electronic supplementary material The online version of this article (https://doi.org/10.1007/s00380-019-01486-y) contains supplementary material, which is available to authorized users.

Takanori Yasu

tyasu@dokkyomed.ac.jp

1 Department of Cardiac and Vascular Surgery, Dokkyo Medical University Nikko Medical Center, Nikko, Tochigi, Japan

2 Department of Cardiac and Vascular Surgery, Dokkyo Medical University, Mibu, Tochigi, Japan

3 Kikuchi Microtechnology Institute, Ryugasaki, Ibaraki, Japan

4 Department of Clinical Laboratory, Dokkyo Medical University Nikko Medical Center, Nikko, Tochigi, Japan

5 National Institute of Advanced Industrial Science and Technology, Tsukuba, Ibaraki, Japan
Abnormal activation states of leukocytes and leukocyte-platelet interactions play key roles in organ injury induced by atherosclerotic disease $[1,2]$, diabetes mellitus, and other inflammatory conditions [3-5]. Leukocyte rheology is critical for modulating microvascular haemodynamics through transformation from resting to active states under

6 Department of Diabetes and Endocrinology, Dokkyo Medical University Nikko Medical Center, Nikko, Tochigi, Japan

7 Department of Rehabilitation, Dokkyo Medical University Nikko Medical Center, Nikko, Tochigi, Japan

8 Department of Cardiovascular Medicine and Nephrology, Dokkyo Medical University Nikko Medical Center, 632 Takatoku, Nikko 321-2593, Tochigi, Japan

9 Department of Cardiology, Dokkyo Medical University Nikko Medical Center, Nikko, Tochigi, Japan 
conditions of inflammation or low shear stress [6-9], such as high-fat diets [4], triggered by free fatty acids [5-7] and ischemia/reperfusion. Decreased leukocyte deformability and increased leukocyte adherence to the post-capillary venular endothelium leads to microvascular dysfunction, in part, through increased blood viscosity [5-7]. Because of its larger volume and higher cellular viscosity, each leukocyte is equivalent to approximately 700 erythrocytes in its tendency to block $5 \mu \mathrm{m}$ capillary channels [8]. The rheology of leukocytes has significant implications on their functional behaviour, including flow-through capillaries and interactions with endothelial cells [5-10].

Cohort studies have shown blood viscosity to be a strong predictor of cardiovascular events [11], particularly in diabetic patients $[12,13]$. However, clinically feasible methods for determining leukocyte activation and the hemorheological character of blood, a non-Newtonian fluid, are limited. A microchannel flow analyser (MCFAN) with a conventional siliconized chip (BK 7-7-4.5D) is a generally accepted ex vivo capillary model for the evaluation of whole blood rheology [5-7, 14-16]. However, the BK 7-7-4.5 model differs from in vivo microvessels in its abrupt narrowing and shorter capillary length. In conventional microarray chips with abrupt narrowing, we have observed platelet aggregation and platelet-leukocyte adhesion at the post-capillary venules and plugging of some terminal capillaries, possibly due to activation of glycoprotein IIb/IIIa on platelets caused by the abrupt increase in shear stress [17]. This artefactual activation of platelets downstream of capillaries may prolong the whole blood passage time somewhat.

Therefore, we designed and manufactured a new microchannel array chip, DKAMCM1-60-7-4.5D, to mimic the human microvessel network. Stepwise increases in shear stress may prevent artefactual activation of platelets. The silicon dioxide film on the silicon wafers provides phase contrast that permits clear microscopic observation of blood cells, especially transparent leukocytes and platelets, from passing through the microchannel array without dying [18].

Although the number of adhesive leukocytes per unit area has been reported as an index of leukocyte activation [5-7], its use is not clinically feasible because the measurement is time-consuming. EDTA-2Na scavenges metal ions $\left(\mathrm{Ca}^{2+}\right.$ and $\left.\mathrm{Mg}^{2+}\right)$ essential to the activation of platelets and some leukocytes $[8,19]$. Therefore, we postulate that the change in passage time between heparinized blood and EDTA-2Na + heparinized blood may reflect leukocyte rheology in whole blood in vivo. To explore clinically feasible indicators of leukocyte activity, as well as blood rheology using MCFAN, with the newly developed siliconized microchannel chip, we examined the correlation between the number of adhesive leukocytes per field of heparinized blood, plasma levels of myeloperoxidase (MPO), and the change of whole blood passage time \{ (heparinized blood) - (EDTA-2Na + heparinized blood) $\}$ under a constant vacuum of $30 \mathrm{cmH}_{2} \mathrm{O}$ and $60 \mathrm{cmH}_{2} \mathrm{O}$ after calibration, according to the duration of saline passage through the channels.

\section{Materials and methods}

\section{Study participants}

The clinical characteristics of the study participants including 79 healthy volunteers, 42 patients with type 2 diabetes mellitus (DM) and 36 patients with acute coronary syndrome (ACS) are shown in Table 1. Seventy-nine healthy, Japanese volunteers did not have any history of DM, hypertension, dyslipidaemia, collagen disease, cardiopulmonary disease, liver and kidney disease, or malignancy; were not currently pregnant or on any medication; and their results of routine physical examinations and standard laboratory tests were normal. Emergent coronary arterial intervention was performed in all the 36 patients with acute coronary syndrome within 10-14 days.

\section{Blood kinetics in an ex vivo microchannel microvascular model}

We used a plot-type microchannel flow analyser system (BWA-MCFAN, Kikuchi Microtechnology Co., Ltd. Ibaraki, Japan) equipped with a new microchannel array chip (DKAMCM1-60-7-4.5D, National Institute of Advanced Industrial Science and Technology, Tsukuba, Ibaraki, Japan) designed as an ex vivo microvascular model to assess whole blood rheology and leukocyte activity. The detailed procedures and apparatus of BWA-MCFAN have been described elsewhere $[14,15]$. In short, microgrooves formed on the surface of a silicon chip were converted to leak-proof microchannels by covering them tightly with an optical flat glass plate in a holder. The contact between the two surfaces could be made watertight by mechanical pressing alone because of their optical flatness. The microgrooves in the silicon microchannel chip were prefilled with saline.

Within 20 min of collecting blood into the two tubes, $0.1 \mathrm{~mL}$ of blood was drawn through the chip under a constant vacuum of $-30 \mathrm{cmH}_{2} \mathrm{O}(2.94 \mathrm{kPa}$, physiological pressure difference between arterioles and venules) or $-60 \mathrm{cmH}_{2} \mathrm{O}(5.88 \mathrm{kPa}$, pathological pressure difference between arterioles and venules). The time required for saline to pass through the microchannels was determined before each blood measurement for calibration. Microscopic motion images of blood passing through microchannels were monitored and stored via computer. When $0.08-0.10 \mathrm{~mL}$ of blood had exited the microchannel array, five fields were recorded, five still images were randomly selected for offline 
Table 1 Clinical characteristics of study subjects

\begin{tabular}{llll}
\hline & $\begin{array}{l}\text { Healthy sub- } \\
\text { jects }(n=79)\end{array}$ & Diabetes mellitus $(n=42)$ & Acute coronary syndrome $(n=36)$ \\
\hline Age (years) & $36.6 \pm 10.9$ & $64.4 \pm 8.5$ & $65.5 \pm 10.1$ \\
Sex (male) & 39 & 30 & 32 \\
Height $(\mathrm{cm})$ & $165.1 \pm 8.6$ & $161.5 \pm 9.4$ & $161.9 \pm 8.0$ \\
Weight $(\mathrm{kg})$ & $61.3 \pm 12.4$ & $70.3 \pm 11.2$ & $64.4 \pm 10.2$ \\
BMI $\left(\mathrm{kg} / \mathrm{m}^{2}\right)$ & $22.4 \pm 3.5$ & $27.1 \pm 4.6$ & $24.5 \pm 3.1$ \\
Current smoker $(\%)$ & $1(1.3 \%)$ & $1(2.3 \%)$ & $14(38.9 \%)$ \\
White blood cells $(/ \mu \mathrm{L})$ & $6462 \pm 1356$ & $6227 \pm 1601$ & $6628 \pm 1908$ \\
Haemoglobin $(\mathrm{g} / \mathrm{dL})$ & $14.0 \pm 1.7$ & $14.2 \pm 1.4$ & $13.3 \pm 1.5$ \\
Haematocrit $(\%)$ & $43.1 \pm 4.3$ & $41.7 \pm 4.2$ & $39.1 \pm 4.1$ \\
Platelets $(10 \% / \mu \mathrm{L})$ & $26.1 \pm 5.0$ & $21.1 \pm 5.2$ & $25.1 \pm 7.7$ \\
LDL cholesterol $(\mathrm{mg} / \mathrm{dL})$ & $102.8 \pm 33.1$ & $86.9 \pm 32.1$ & $84.2 \pm 24.6$ \\
HDL cholesterol $(\mathrm{mg} / \mathrm{dL})$ & $60.9 \pm 18.3$ & $49.5 \pm 11.1$ & $39.1 \pm 13.0$ \\
Triglycerides $(\mathrm{mg} / \mathrm{dL})$ & $94.5 \pm 83.7$ & $150.8 \pm 88.9$ & $138.9 \pm 80.2$ \\
Plasma glucose $(\mathrm{mg} / \mathrm{dL})$ & $87.7 \pm 13.2$ & $157.0 \pm 52.0$ & $133.7 \pm 58.7$ \\
eGF min R (mL//1.73) & $91.6 \pm 18.7$ & $69.7 \pm 18.4$ & $62.3 \pm 17.7$ \\
HbA1c $(\%)$ & $5.2 \pm 0.3$ & $7.9 \pm 1.0$ & $6.7 \pm 1.7$ \\
BNP $(\mathrm{pg} / \mathrm{mL})$ & & $32.5 \pm 39.0($ median 11.6$)$ & $71.0 \pm 50.8(\mathrm{median} 55.8)$ \\
\hline
\end{tabular}

Data are expressed as the mean $\pm \mathrm{SD}$

$B M I$ body mass index, $L D L$ low-density lipoprotein, $H D L$ high-density lipoprotein, $e G F R$ estimated glomerular filtration rate, $H b A l c$ haemoglobin A1c, $S D$ standard deviation, $B N P$ brain natriuretic peptide analysis, and the numbers of adhesive or clumped leukocytes on the microchannel platform in these images were counted [5-7] by middle-powered field $(\times 700)$. Adhesive leukocytes were defined as static leukocytes with a clear surface border on still images [5-7]. The whole blood passage time was normalized as follows: Normalized whole blood passage time $=$ passage time of $100 \mu \mathrm{L}$ of blood $(\mathrm{sec}) * \times 12 \mathrm{~s} /$ passage time of $100 \mu \mathrm{L}$ of saline (s). Plasma levels of MPO were determined using an enzyme-linked immunosorbent assay (ELISA) kit (Bio Check, Inc., Foster City, CA, USA) [6].

\section{Study protocol}

The study protocol was approved by the Ethical Committee of Dokkyo Medical University Nikko Medical Center. All procedures performed in this study were conducted with informed consent of the patients and complied with the national ethical guidelines for medical and health research involving human subjects and with the 1964 Helsinki Declaration and its later amendments or comparable ethical standards.

All participants arrived in the laboratory at 2:00 pm. The participants had abstained from alcohol, caffeine, and smoking for at least $12 \mathrm{~h}$ prior to the study. All participants drank
$200 \mathrm{~mL}$ of water and remained seated at rest for $5 \mathrm{~min}$ before blood sampling. Blood $(10 \mathrm{~mL})$ was sampled from the antecubital vein, and $5 \mathrm{~mL}$ was collected into each of two tubes: a $5 \%$ vol heparin tube (Venoject II, Terumo, Tokyo, Japan) and an EDTA-2Na + 5\% vol heparin tube (Venoject II, Terumo, Tokyo, Japan). FMLP (Sigma-Aldrich, Tokyo, Japan) was used to induce leukocyte activation experimentally $[19,20]$. FMLP is a chemotactic peptide derived from bacterial protein degradation and mitochondrial proteins upon tissue damage [21, 22] and is present in low concentrations in the bloodstream during inflammation [21, 22]. FMLP was dissolved in dimethyl sulfoxide (Sigma-Aldrich). FMLP $\left(10^{-9}\right.$ or $\left.10^{-10} \mathrm{M}\right)$ was added into whole blood from healthy subjects $(n=10)$ in the heparinized tube and EDTA-2Na + heparin tube. After 5 min incubation with FMLP, the whole blood passage time was measured by BWA-MCFAN.

\section{Statistical analysis}

Data were statistically analysed using JMP $14.0 \mathrm{~J}$ software (SAS Institute, Cary, NC, USA). Continuous variables are described as the mean \pm standard deviation (SD). Differences were analysed with Student's $t$ test or 2-way analysis of variance. Correlations were assessed using Fisher's coefficient $(r)$. A $p$ value $<0.05$ was considered statistically significant. 


\section{Results}

The pharmacological mechanisms of two commonly used anti-coagulants, heparin and ethylene-diamine-tetraacetic acid (EDTA)-2Na, are demonstrated in whole blood ex vivo (Fig. 1). A blueprint of the new microchannel array (DKAMCM1-60-7-4.5D, National Institute of Advanced Industrial Science and Technology, Tsukuba, Ibaraki, Japan) designed to mimic the human microvessel network employed in this work, is shown in Fig. 2. The designed pattern was machined onto a photomask blank, and an i-line stepper (Model NSR-2205i12D, Nikon, Tokyo, Japan) was used to transfer the photomask pattern onto photoresist, coated onto 4-in. silicon wafers (Fig. 3a, b, e). After the development of the photoresist, an inductively coupled plasma dry-etching system (Model RIE-101iPHSL, SAMCO, Tokyo, Japan) was used to pattern the silicon wafers with sulphur hexafluoride etchant gas (Fig. 3e, f). To set up the test blood supply and recovery tubes in the silicon chips, holes with a $1.5 \mathrm{~mm}$ diameter were machined through the silicon wafers by maskless photolithography (Model DL-1000, Nano System Solutions, Inc., Okinawa, Japan) and the inductively coupled plasma dry-etching system described above. Diluted KOH solution was used to slightly etch the patterned silicon to obtain a smoother surface (Fig. 3i, j). To form a blood-inert surface, the wafers were then introduced into a plasma chemicalvapor deposition system (Model PD-20SS, SAMCO), and a silicon dioxide film with a thickness of $0.4-1.0 \mu \mathrm{m}$ was deposited at $350{ }^{\circ} \mathrm{C}$ by a mixture of tetraethoxysilane and oxygen (Fig. 3h). This film, on the silicon wafers, provided phase contrast that facilitated clear microscopic observation of blood cells, especially transparent leukocytes and platelets. The wafers were then cut into pieces of $8 \times 16 \mathrm{~mm}^{2}$ each using a dicing saw (Model DAD522, DISCO, Tokyo, Japan) to obtain the microchannel chips. The resulting pattern was carefully examined with a scanning electron microscope (Model S-4800, Hitachi Hightechnologies, Tokyo, Japan) (Fig. 3k) and confocal laser microscope (Model LEXT OLS4100, Olympus Corp., Tokyo, Japan) (Fig. 31). Schematic of leukocytes passing through capillaries from the above and from the side are shown in Fig. 3m, n, respectively.

First, we determined whether our new BWA-MCFANequipped method with DKAMCM1-60-7-4.5D could quantify leukocyte activation induced by $N$-formyl-methionylleucyl-phenylalanine (FMLP) ex vivo. FMLP was added to fresh whole blood from healthy subjects to activate leukocytes experimentally [19, 20], and then the whole blood passage time was measured by BWA-MCFAN. The addition of FMLP to heparinized blood significantly increased the whole blood passage time and adhesive leukocyte number, which were significantly correlated (Fig. 4a). However, the whole blood passage time and adhesive leukocytes in blood treated with EDTA-2Na and heparin only slightly increased after FMLP application (Fig. 4b).

Next, we confirmed that passage times of whole blood with heparin +EDTA-2Na were always shorter than those of whole blood with only heparin in healthy subjects $(n=79$, Table 1) under both suction pressures of $-30 \mathrm{cmH}_{2} \mathrm{O}$ and $-60 \mathrm{cmH}_{2} \mathrm{O}$ (Fig. 4c, d, Videos 1, 2). There were variations in the number of adhesive leukocytes per field in heparinized blood below $-30 \mathrm{cmH}_{2} \mathrm{O}$ (Fig. 4e) and fewer below $-60 \mathrm{cmH}_{2} \mathrm{O}$ (Fig. 4f). Numbers of adhesive leukocytes in blood with heparin +EDTA-2Na were remarkably lower than

\section{Different pharmacological mechanisms of heparin and EDTA-2 $\mathrm{Na}$ in whole blood in ex vivo}

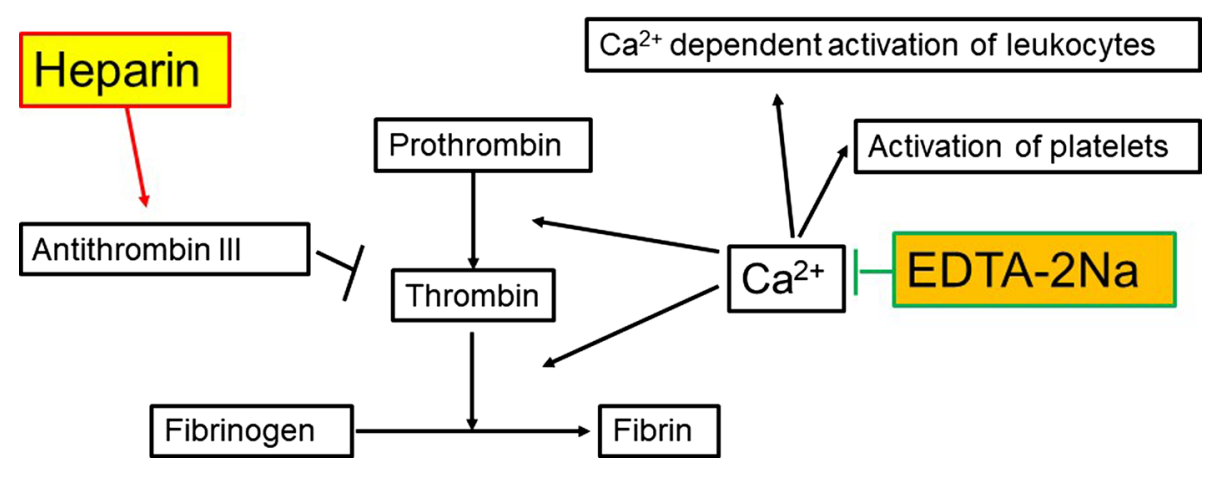

Fig. 1 Different pharmacological mechanisms of heparin and ethylene-diamine-tetraacetic acid (EDTA)-2Na in whole blood ex vivo. Pharmacological mechanisms of heparin and EDTA-2Na in whole blood ex vivo are demonstrated in this schematic. Heparin binds to the enzyme inhibitor antithrombin (AT) III and greatly accelerates the rate at which AT III inactivates the coagulation enzymes thrombin and factor $\mathrm{Xa}$. In contrast, EDTA-2Na scavenges metal ions $\left(\mathrm{Ca}^{2+}\right.$ and $\left.\mathrm{Mg}^{2+}\right)$, thereby inhibiting activation of platelets and $\mathrm{Ca}^{2+}$ dependent activation of leukocytes. The heparinized blood tube maintained leukocyte and platelet function ex vivo, just as it did in vivo 
a Blueprint of new designed microchannel array

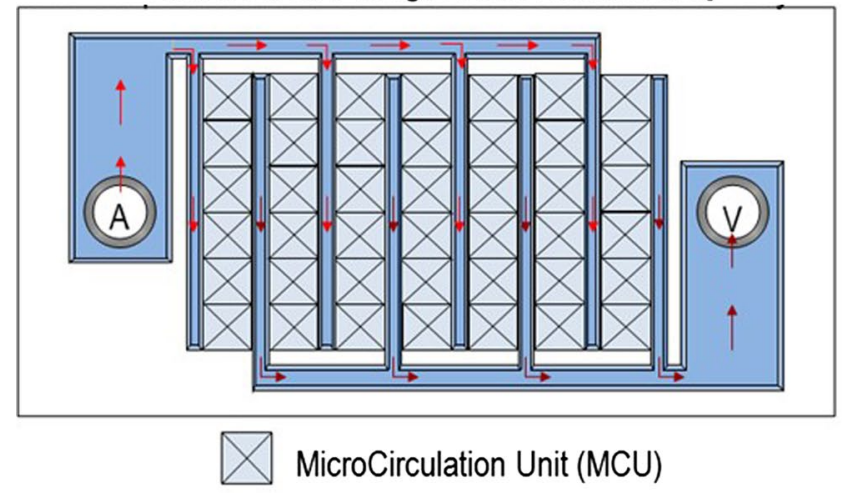

b Capillary Array Unit (CAU)

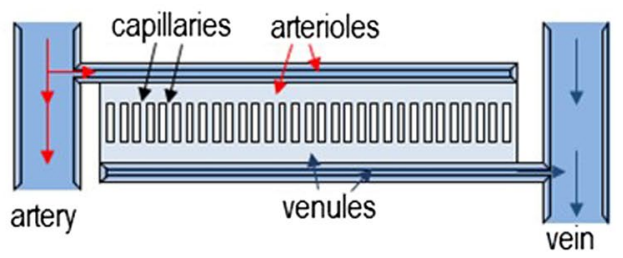

C

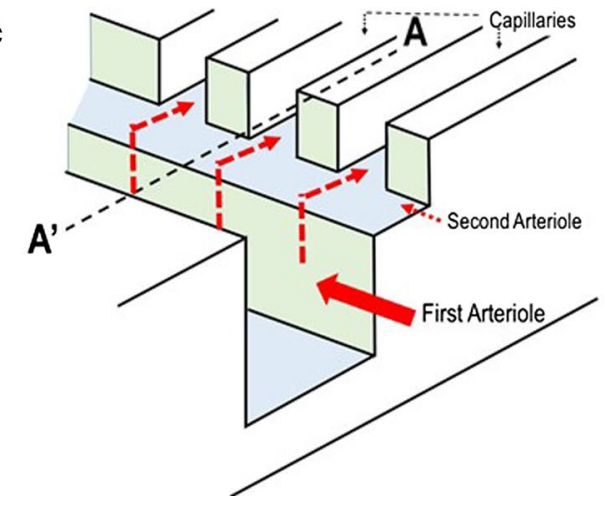

Fig. 2 Blueprint of new microchannel array (DKAMCM1-60-74.5D) designed to mimic the human microvessel network. a Blood sample enters from hole A (artery) into the microchannel array and exits by hole V (vein). b A capillary array unit (CAU) comprises 40 capillaries (black arrows) with length, width, and depth of 60, 7, and $4.5 \mu \mathrm{m}$, respectively, and each capillary is connected to an arteriole (red arrows) and venule (blue arrows) with length, width, and depth of 640,30 , and 4.5 (light blue) or 50 (blue) $\mu \mathrm{m}$, respectively. c Threedimensional blueprint of connection of primary arterioles (wide red arrow, $50 \mu \mathrm{m}$ depth) to secondary arterioles (narrow dashed red lines, $4.5 \mu \mathrm{m}$ depth), and then to capillaries (narrow red arrows, $4.5-\mu \mathrm{m}$ depth). The blood sample flows through primary arterioles, secondary arterioles, and capillaries following the red arrows. d A microcirculation unit (MCU) comprising four CAUs. a, e The 42 MCUs are connected via arteries (red arrows) and veins (dark blue arrows) with a depth of $50 \mu \mathrm{m}$. Blood sample enters from the artery into MCUs and exits via the vein

tube) $-($ EDTA-2Na + heparin) $\}$ and adhesive leukocyte number (heparin) under $-30 \mathrm{cmH}_{2} \mathrm{O}(r=0.50, p<0.0001)$ (Fig. 5f). There was also a significant correlation between change in whole blood passage time and plasma levels of MPO ( $r=0.66, p<0.0001)$ (Fig. 5g).

Additionally, we measured the passage times of whole blood with heparin and whole blood with heparin + EDTA$2 \mathrm{Na}$ under a constant vacuum of $25 \mathrm{cmH}_{2} \mathrm{O}, 30 \mathrm{cmH}_{2} \mathrm{O}$, or $35 \mathrm{cmH}_{2} \mathrm{O}$ in 25 subjects to determine the best pressure for assessing leukocyte activity (Fig. 6). The passage time of whole blood with heparin under a constant vacuum of $25 \mathrm{cmH}_{2} \mathrm{O}$ could not be measured in 19 of 25 subjects because of obstruction of many microchannel arrays by activated leukocytes and platelets. The optimal pressure for assessing leukocyte activity may be $30 \mathrm{cmH}_{2} \mathrm{O}$ and/ or $35 \mathrm{cmH}_{2} \mathrm{O}$. 


\section{a Silicon wafer}

$\mathrm{Si}$

e Photolithography

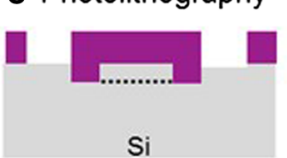

b Photolithography

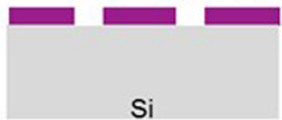

f A $50 \mu \mathrm{m}$ Deep RIE

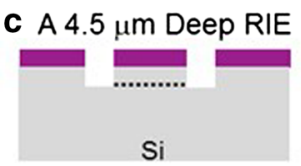

g Resist removal

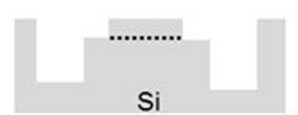

$\mathrm{Si}$

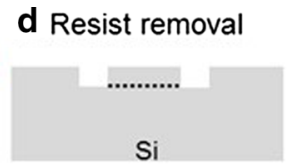

h $\mathrm{SiO}_{2}(\mathrm{PECVD})$

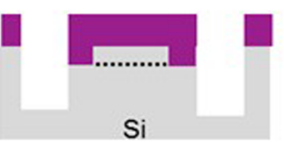

$\mathrm{Si}$

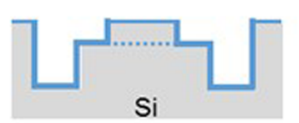

$\mathrm{Si}$
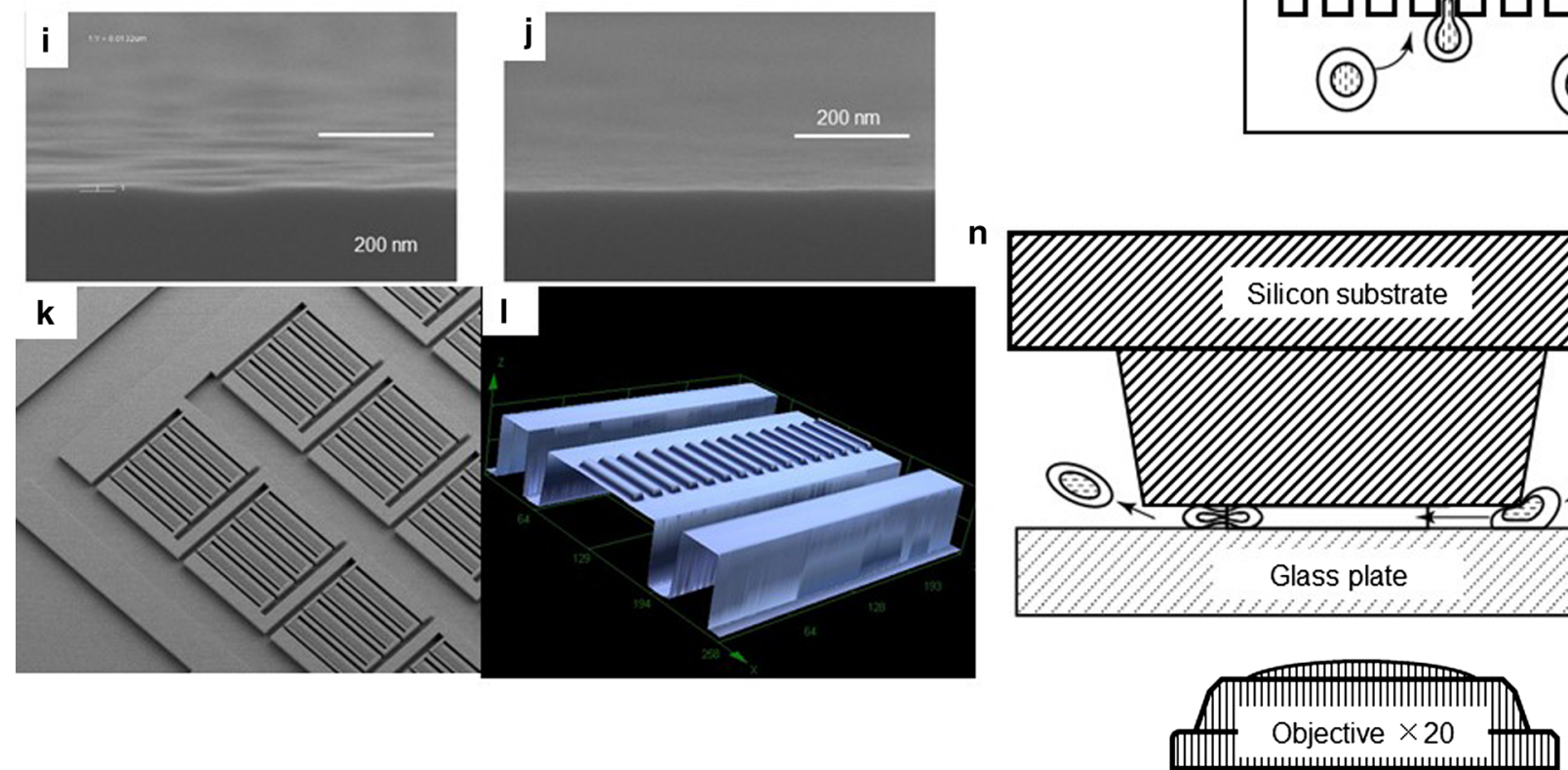

Fig. 3 Fabrication process for the microchannel array. a, b, e The designed pattern was machined onto a photomask blank, and an $i$-line stepper was used to transfer the photomask pattern onto photoresistcoated 4-in. silicon wafers. c, f After the development of the photoresist, inductively coupled plasma dry-etching was used to pattern the silicon wafers with sulphur hexafluoride etchant gas. $\mathrm{g}$ Diluted potassium hydroxide $(\mathrm{KOH})$ solution was used to slightly etch the patterned silicon to obtain a smoother surface after resist removal. h After fabrication, 1- $\mu$ f-thick silicon dioxide $\left(\mathrm{SiO}_{2}\right)$ was deposited

\section{Discussion}

We developed a clinically feasible method for assessing leukocyte rheology in whole blood using a MCFAN with a novel silicon chip designed to mimic the human microvascular network. We designed and developed a new multi-manifold microchannel array chip, DKAMCM1-60$7-4.5 \mathrm{D}$, using dry-etching, resulting in a square valley to observe how blood cells pass through the capillary tube when they are deformed. The silicon dioxide film on the silicon wafers provided both a blood-inert surface and phase contrast for microscopic observation of moving blood cells [18], even erythrocytes and leukocytes transiently deformed at the capillaries, agglomerated in the in deep trenches by plasma-enhanced chemical-vapor deposition (PECVD). $\mathbf{i}, \mathbf{j}$ The bottom of a $50 \mu \mathrm{m}$ trench was imaged by scanning electron microscopy (SEM) before $\mathbf{i}$ and after $\mathbf{j}$ slight etching by diluted $\mathrm{KOH}$ solution. $\mathbf{k}, \mathbf{l}$ Microchannel array was carefully examined by SEM (k) and by confocal laser microscopy (l). $\mathbf{m}, \mathbf{n}$ Erythrocytes and leukocytes, which are larger and more viscous than erythrocytes, are transiently deformed at the capillaries. Schematic of leukocytes passing through capillaries from above (m) and from the side $(\mathbf{n})$ are shown

capillaries, or adhered to post-capillary venules (Videos 1,2).

In addition, the present new microchannel array chip prevents artefactual activation of platelets by a stepwise decrease in microchannel diameter (Video 1). This artefactual activation of platelets downstream of capillaries induced by conventional microarray chips with abrupt narrowing may prolong to the whole blood passage time somewhat [17].

In the presence of $\mathrm{Ca}^{2+}$, leukocytes can transform to an active state with pseudopods and increased viscoelastic coefficients [7, 19, 20, 23, 24]. FMLP increases $\mathrm{Ca}^{2+}$ levels in human neutrophils in a concentration-dependent manner, as determined by Fura- 2 imaging and reactive oxygen 

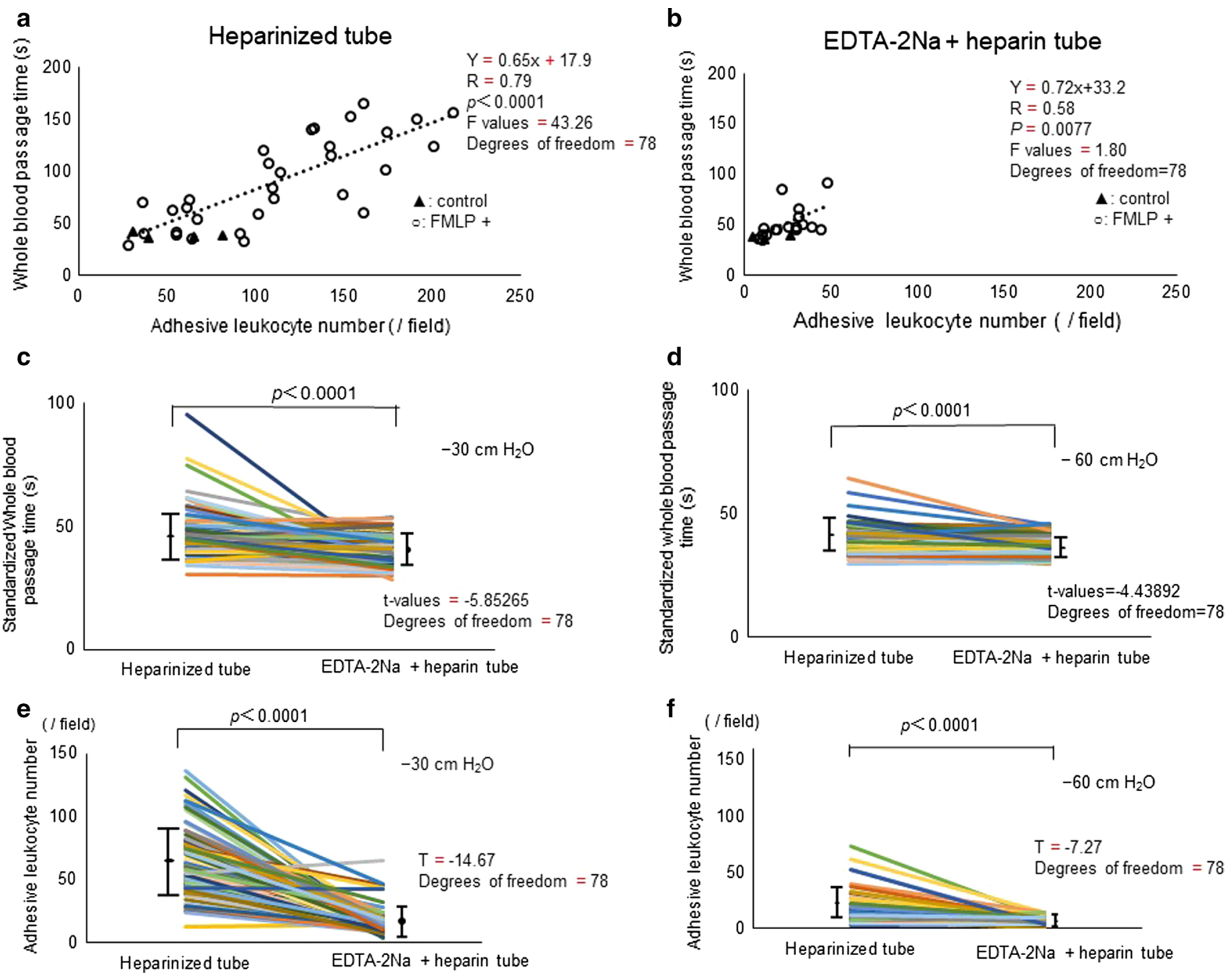

Fig. 4 Whole blood passage time through microchannel arrays and adhesive leukocytes. a, b There was a significant correlation between passage time of heparinized whole blood through microchannel arrays and adhesive leukocyte number per field (a $r=0.79$, $p<0.0001)$ and those of ethylene-diamine-tetraacetic acid (EDTA)$2 \mathrm{Na}+$ heparinized blood (b $r=0.58 p=0.0077$ ) with (filled triangle)/without (open circle) $10^{-9}$ or $10^{-10} \mathrm{M}$ formyl-methionyl-leucylphenylalanine (FMLP) to activate leukocytes. FMLP significantly increased the numbers of adherent or clumped leukocytes and sig-

intermediates production [20]. In the present study, application of FMLP to heparinized whole blood in the presence of $\mathrm{Ca}^{2+}$ significantly increased the adhesive leukocyte number, and EDTA-2Na inhibited FMLP-induced activation of leukocytes and platelets by scavenging $\mathrm{Ca}^{2+}$. EDTA inhibits leukocyte activation by (1) inhibition of $\mathrm{Ca}^{2+}$-dependent activation of leukocytes, and (2) inhibition of platelet-leukocyte interaction. The inhibition of activation of leukocytes and platelets by EDTA-2Na was associated with an increase in leukocyte deformability and a decrease in leukocyte adhesion to the chip and platelets. The change in the passage time of whole blood, which is significantly correlated with nificantly increased the duration of whole blood passage. c, $\mathbf{d}$ Standardized passage time of whole blood with heparin and blood with heparin + EDTA-2Na under constant vacuum of $30 \mathrm{cmH}_{2} \mathrm{O}$ and $60 \mathrm{cmH}_{2} \mathrm{O}$. Note that the passage time of whole blood with heparin +EDTA-2Na was significantly shorter $(p<0.0001)$ than that of whole blood with only heparin under both suction pressures. e, f The adhesive leukocyte number (/field) in blood with heparin +EDTA$2 \mathrm{Na}$ was remarkably less than that in blood with heparin alone under both suction pressures $(p<0.0001)$

the adhesive leukocyte number and plasma levels of MPO, was calculated using BWA-MCFAN by subtraction of the passage time of whole blood treated with EDTA-2Na + heparin from that of blood treated with heparin only, even in blood from patients with DM and ACS, who suffered from inflammation. This technique may provide a clinically feasible method for determination of leukocyte rheology. The rheology of leukocytes has significant implications for their functional behaviour, including flow through, microcirculation, and interaction with endothelial cells [24-26]. The optimal pressure for assessing leukocyte activity may be $30 \mathrm{cmH}_{2} \mathrm{O}$ and/or $35 \mathrm{cmH}_{2} \mathrm{O}$. 


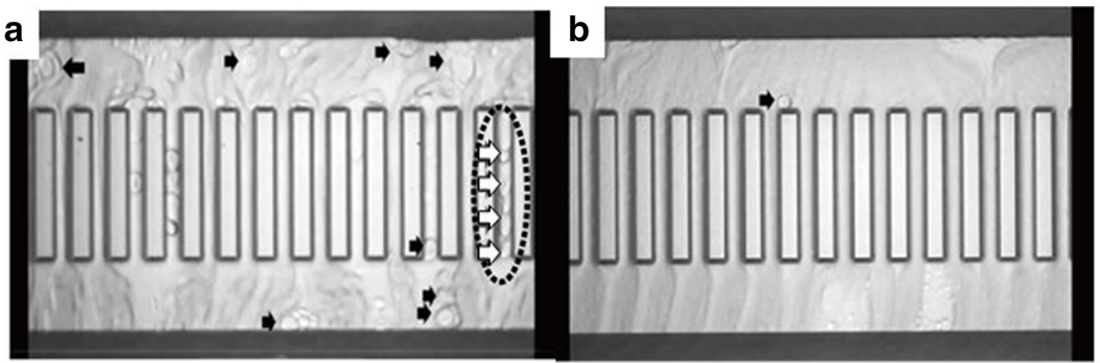

C Adhesive leukocyte number

d

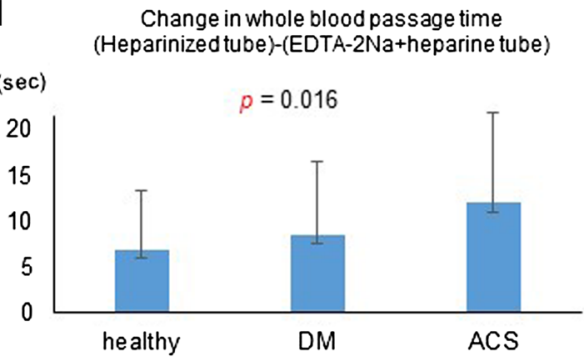

e

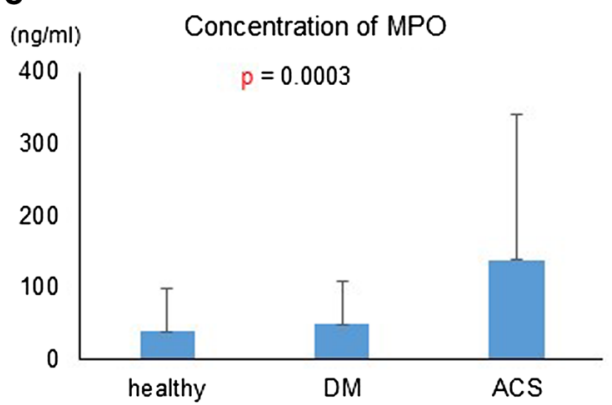

f

Correlation between adhesive leukocyte number (field) in heparinized tube and change in whole blood passage time (heparinized tube) (EDTA-2Na + heparin tube)
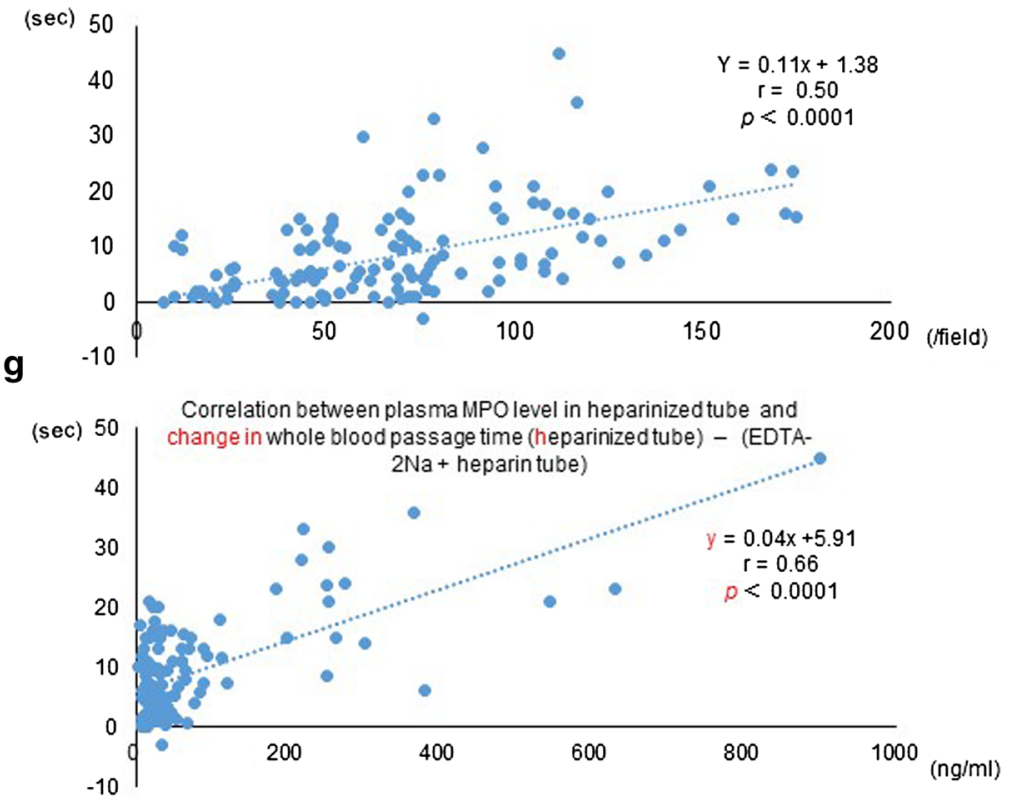

Fig. 5 Microphotographs of whole blood passing through microchannel arrays Blood was sampled from the same healthy subject (a, b). a Adherent or clumped leukocytes (black arrows) were observed in whole blood with heparin in the microchannel flow analyser (MCFAN) by high-powered field $(\times 1400)$. Deformed erythrocytes running through capillaries (white open arrows) were visible in the high-powered field (a). b There was no platelet aggregation and significantly fewer adherent leukocytes (black arrow) in blood with ethylene-diamine-tetraacetic acid (EDTA)-2Na + heparin because of $\mathrm{Ca}^{2+}$ scavenging by EDTA-2Na. c The number of adhesive leukocytes was significantly lower $(p<0.0001)$ in healthy subjects $(n=79)$

In conclusion, we have developed a clinically feasible method for assessing leukocyte rheology in whole blood using BWA-MCFAN with a new silicon chip designed to mimic the human microvascular network. Using the anticoagulants heparin and EDTA-2Na, we were able to than those subjects with diabetes mellitus (DM) $(n=42)$ and acute coronary syndrome (ACS) $(n=36)$. $\mathbf{d}$ Change in whole blood passage time (heparinized tube) $-($ EDTA-2Na + heparin tube) in patients with DM and ACS were increased compared samples from healthy subjects. e Plasma concentration of myeloperoxidase (MPO) of healthy subjects was lower than those of DM and ACS subjects. $\mathbf{f}$ There was a significant correlation between change in whole blood passage time $\{($ heparin tube $)-($ EDTA-2Na + heparin $)\}$ and adhesive leukocyte number (heparin) under $-30 \mathrm{cmH}_{2} \mathrm{O}(r=0.50, p<0.0001)$. g There was also a significant correlation between change in whole blood passage time and plasma levels of MPO $(r=0.66, p<0.0001)$ quantify leukocyte rheology and show that the change in whole blood passage time, as calculated by subtraction of the passage time of blood treated with both heparin and EDTA-2Na from that of blood treated with heparin only, is correlated with adhesive leukocyte number. 


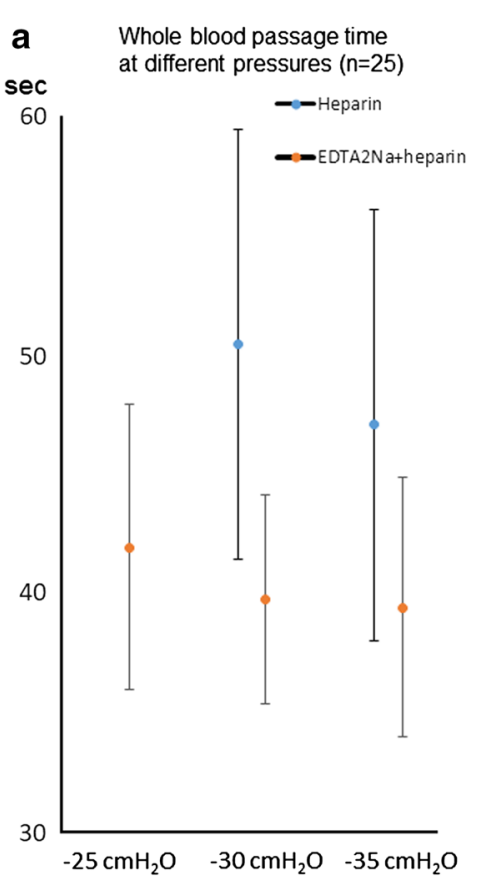

b

sec heparinized tube and change in whole blood passage time

50

30

20

10

0

$-10^{0}$

c

sec Correlation between adhesive leukocyte number (ifield) in

50 heparinized tube and change in whole blood passage time

(heparinized tube) - (EDTA-2Na + heparin tube) $(n=25)$

$40-35 \mathrm{cmH}_{2} \mathrm{O}$

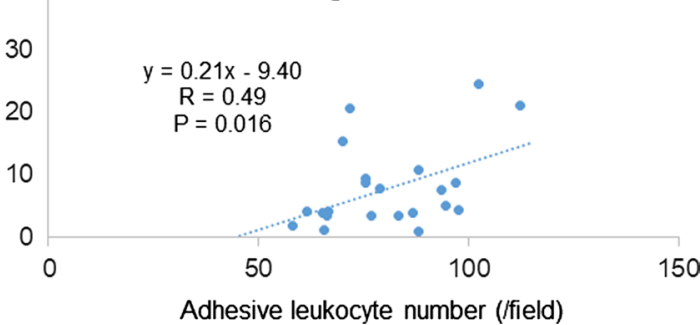

Fig. 6 Passage times of whole blood through microchannel arrays under $-25 \mathrm{cmH}_{2} \mathrm{O},-30 \mathrm{cmH}_{2} \mathrm{O}$, and $-35 \mathrm{cmH}_{2} \mathrm{O}$. Standardized passage times of whole blood with heparin and whole blood with heparin + EDTA-2Na under a constant vacuum of $25 \mathrm{cmH}_{2} \mathrm{O}$, $30 \mathrm{cmH}_{2} \mathrm{O}$, or $35 \mathrm{cmH}_{2} \mathrm{O}$ in 25 subjects (a). The passage time of whole blood with heparin under a constant vacuum of $25 \mathrm{cmH}_{2} \mathrm{O}$ could not be measured in 19 of 25 subjects because of obstruction of the microchannel arrays. The passage time of whole blood with heparin + EDTA-2Na was shorter than that of whole blood with heparin under both $30 \mathrm{cmH}_{2} \mathrm{O}$ and $35 \mathrm{cmH}_{2} \mathrm{O}$ suction pressures. There was a significant correlation between change in whole blood passage time \{(heparin) $-($ EDTA-2Na + heparin $)\}$ and adhesive leukocyte number (heparin) under $-30 \mathrm{cmH}_{2} \mathrm{O}(\mathbf{b} r=0.44, p=0.029)$ and $-35 \mathrm{cmH}_{2} \mathrm{O}$ (c $r=0.49, p=0.016$ )

Ethical statement All procedures performed in this study were conducted with the subjects' informed consent, and complied with the national ethical guidelines for medical and health research involving human subjects and with the 1964 Helsinki declaration and its later amendments or comparable ethical standards.

Open Access This article is distributed under the terms of the Creative Commons Attribution 4.0 International License (http://creativeco mmons.org/licenses/by/4.0/), which permits unrestricted use, distribution, and reproduction in any medium, provided you give appropriate credit to the original author(s) and the source, provide a link to the Creative Commons license, and indicate if changes were made.

\section{References}

1. Ross R (1990s) The pathogenesis of atherosclerosis: a perspective for the 1990s. Nature 362:801-809

2. Garcia-Prieto J, Villena-Gutiérrez R, Gómez M, Bernardo E, Pun-García A, García-Lunar I, Crainiciuc G, Fernández-Jiménez R, Sreeramkumar V, Bourio-Martínez R, García-Ruiz JM, Del Valle AS, Sanz-Rosa D, Pizarro G, Fernández-Ortiz A, Hidalgo A, Fuster V, Ibanez B (2017) Neutrophil stunning by metoprolol reduces infarct size. Nat Commun 8:14780 
3. Preira $P$, Forel JM, Robert $P$, Nègre $P$, Biarnes-Pelicot $M$, Xeridat F, Bonqrand P, Papazian L, Theodoly O (2016) The leukocytestiffening property of plasma in early acute respiratory distress syndrome (ARDS) revealed by a microfluidic single-cell study: the role of cytokines and protection with antibodies. Crit Care 20:8

4. Nishimura S, Manabe I, Nagasaki M, Seo K, Yamashita H, Hosoya Y, Ohsugi M, Tobe K, Kadowaki T, Nagai R, Sugiura S (2008) In vivo imaging in mice reveals local cell dynamics and inflammation in obese adipose tissue. J Clin Invest 118:710-721

5. Azekoshi Y, Yasu T, Watanabe S, Tagawa T, Abe S, Yamakawa K, Uehara Y, Momomura S, Urata H, Ueda S (2010) Free fatty acid causes leukocyte activation and resultant endothelial dysfunction through enhanced angiotensin II production in mononuclear and polymorphonuclear cells. Hypertension 56:136-142

6. Yasu T, Kobayashi M, Mutoh A, Yamakawa K, Momomura S, Ueda S (2013) Dihydropyridine calcium channel blockers inhibit non-esterified-fatty-acid-induced endothelial and rheological dysfunction. Clin Sci 125:247-255

7. Yasu T, Mutoh Wada H, Kobayashi M, Kikuchi Y, Momomura S, Ueda S (2018) Renin-angiotensin system inhibitors can prevent intravenous lipid infusion-induced myocardial microvascular dysfunction and leukocyte activation. Circ J 82:494-501

8. Chien S, Sung KL, Schmid-Schönbein GW, Skalak R, Schmalzer EA, Usami S (1987) Rheology of leukocytes. Ann N Y Acad Sci 516:333-347

9. Fukuda S, Yasu T, Predescu DN, Schmid-Schönbein GW (2000) Mechanisms for regulation of fluid shear stress response in circulating leukocytes. Circ Res 86:E13-E18

10. Lipowsky HH (2005) Microvascular rheology and hemodynamics. Microcirculation 12:5-15

11. Lowe GD, Lee AJ, Rumley A, Price JF, Fowkes FG (1997) Blood viscosity and risk of cardiovascular events: the Edinburgh Artery Study. Br J Haematol 96:168-173

12. Tamariz LJ, Young JH, Pankow JS, Yeh HC, Schmidt MI, Astor B, Brancati FL (2008) Blood viscosity and hematocrit as risk factors for type 2 diabetes mellitus: the Atherosclerosis Risk in Communities (ARIC) Study. Am J Epidemiol 168:1153-1160

13. Jax TW, Peters AJ, Plehn G, Schoebel FC (2009) Hemostatic risk factors in patients with coronary artery disease and type 2 diabetes-a two year follow-up of 243 patients. Cardiovasc Diabetol $8: 48$

14. Kikuchi Y, Sato K, Ohki H, Kaneko T (1992) Optically accessible microchannels formed in a single-crystal silicon substrate for studies of blood rheology. Microvasc Res 44:226-240

15. Kikuchi Y, Sato K, Mizuguchi Y (1994) Modified cell-flow microchannels in a single-crystal silicon substrate and flow behavior of blood cells. Microvasc Res 47:126-139
16. Fukuda S, Yasu T, Kobayashi N, Ikeda N, Schmid-Shönbein GW (2004) Contribution of fluid shear response in leukocytes to hemodynamic resistance in the spontaneously hypertensive rat. Circ Res 95:100-108

17. Holme PA, Orvim U, Hamers MJ, Solum NO, Brosstad FR, Barstad RM, Sakariassen KS (1997) Shear-induced platelet activation and platelet microparticle formation at blood flow conditions as in arteries with a severe stenosis. Arterioscler Thromb Vasc Biol 17:646-653

18. Jenison R, Yang S, Haeberli A, Polisky B (2001) Interferencebased detection of nucleic acid targets on optically coated silicon. Nat Biotechnol 19:62-65

19. Kitagawa S, Takaku F, Sakamoto S (1980) A comparison of the superoxide-releasing response in human polymorphonuclear leukocytes and monocytes. J Immunol 125:359-364

20. Chen LW, Jan CR (2001) Mechanisms and modulation of formylmethionyl-leucyl-phenylalanine (fMLP)-induced $\mathrm{Ca}^{2+}$ mobilization in human neutrophils. Int Immunopharmacol 1:1341-1349

21. Anton P, O'Connell J, O'Connell D, Whitaker L, O'Sullivan GC, Collins JK, Shanahan F (1998) Mucosal subepithelial binding sites for the bacterial chemotactic peptide, formyl-methionylleucyl-phenylalanine (FMLP). Gut 42:374-379

22. Marasco WA, Phan SH, Krutzsch H, Showell HJ, Feltner DE, Naim R, Becker EL, Ward PA (1984) Purification and identification of formyl-methionyl-leucyl-phenylalanine as the major peptide neutrophil chemotactic factor produced by Escherichia coli. J Biol Chem 259:5430-5439

23. Feske S (2007) Calcium signalling in lymphocyte activation and disease. Nat Rev Immunol 7:690-702

24. Zhang S, Zheng Y, Chen S, Huang S, Liu K, Lv Q, Jiang Y, Yuan Y (2016) Suilysin-induced platelet-neutrophil complexes formation is triggered by pore formation-dependent calcium influx. Sci Rep 6:36787

25. Boneschansker L, Inoue Y, Oklu R, Irimia D (2016) Capillary plexuses are vulnerable to neutrophil extracellular traps. Integr Biol (Camb) 8:149-155

26. Yasu T, Ikeda N, Ishizuka N, Matsuda E, Kawakami M, Kuroki M, Imai N, Ueba H, Fukuda S, Schmid-Shönbein GW, Saito M (2002) Nicorandil and leukocyte activation. J Cardiovasc Pharmacol 40:684-692

Publisher's Note Springer Nature remains neutral with regard to jurisdictional claims in published maps and institutional affiliations. 